\title{
Human Activity Recognition for Physical Rehabilitation Using Wearable Sensors Fusion and Artificial Neural Networks
}

\author{
Eliasz Kańtoch ${ }^{1}$ \\ ${ }^{1}$ AGH University of Science and Technology, Kraków, Poland
}

\begin{abstract}
Physical inactivity has become one of the leading cause of death worldwide. Sedentary lifestyle is associated with an increased risk of morbidity including cardiovascular diseases, hypertension, cancer, obesity or type 2 diabetes. However, accurate exercise tracking has not been fully resolved yet. The main aim of the study was to create computable algorithm which could be implemented into wearable sensor system to help its users tracking squats as a common rehabilitation exercise. The prototype of battery-operated wearable health tracking device which tracks body temperature and body motions was developed. Pulse oximeter sensor was used to track heart rate. Seven healthy volunteers were recruited into the study. Each volunteer was asked to attach wearable device to the lower chest using elastic belt and perform activities of daily living (ADL) including sit, walk, stand and squats. All study data were recorded. The Multilayer Perceptron topology with hidden neurons was used for classification of squats. The experimental results demonstrated that the method achieved a total accuracy of $82 \%$, sensitivity of $85 \%$ and specificity of $90 \%$.
\end{abstract}

\section{Introduction}

Physical inactivity has become one of the leading cause of death worldwide. Sedentary lifestyle is associated with an increased risk of morbidity including cardiovascular diseases, hypertension, cancer, obesity or type 2 diabetes. There are the number of the off-the-shelves fitness trackers and smartwatches that motivates users to move more by tracking steps, distance, calories, sleep quality or heart rate. However, accurate selected exercise detection in free living environment has not been fully resolved yet.

Recent advances in wearable sensing technologies allow to use mobile sensors in a range of environments. The major advantage of those technologies is their ability for long-term continuous recording of the patient activities in a free-living environment. The other emerging application are smart homes solutions that acquire more and more clinically significant data from the human environment. Context-aware posture monitoring systems can be developed to help in understanding the progression of disease, assess the treatment effectiveness and supervise rehabilitation processes. An activity monitoring system deployed in the patient home may therefore be a crucial step in future care giving. Human's activity is recognized mostly based on the signals acquired from multiple onbody sensors or cameras.

Lara et al. [1] published survey of human activity recognition based on wearable sensors. Twenty eight systems were qualitatively compared in regards to recognition accuracy, response time and other design issues. The majority of system was based on accelerometers and the accuracy rate oscillated between $77 \%$ and $97 \%$ depending on the activities. Kasteren et al. [2] presented in-home activity monitoring system for elderly care that consists of a wireless sensor network and a recognition model. It was proved that differences in the layout of houses and the way a dataset was annotated can affect the performance in activity recognition. Del Din et. al. [3] quantified clinically relevant gait characteristics organized in five domains using laboratory based and freeliving data collected over 7 days using body worn devices placed on the lower back in Parkinson disease participants. The study showed that gait characteristics measured in free-living conditions were significantly different between controls and people with PD compared to two measured in laboratory. Reddy S. et al. [4] developed classification system that uses a mobile phone with a build-in GPS receiver and an accelerometer to identify whether an individual is stationary, walking, running, biking or in motorized transport. Past studies of our AGH Wearables Research Group focused on wireless health monitoring systems using dedicated ECG sensors and movements' sensors placed on the human body [5], telemedical health monitoring system [6], turning domestic appliances into a sensor network for monitoring of activities of daily living [7] and BAN-based monitoring system for in-home applications based on both wearable and stationary sensors to monitor human physiological and movement parameters [8]. The aim of this study was to create computable algorithm which could be implemented into wearable sensor system to help its users tracking squats as a common physical rehabilitation exercise.

This paper is organized as follows: Section 2 describes the developed device and methodology. Section 3 describes the experiments, datasets and results for evaluating our approach. Finally, Section 4 sums up our conclusions. 


\section{Methodology}

In this section we described the hardware used in this study, and the architecture of the system for activity recognition.We developed the prototype of batteryoperated Wearable Health Tracking Device (WHTD) which tracks body temperature and body motions. We used the InvenSense 6-axis Montion Tracking device that combines a 3-axis gyroscope, 3-axis accelerometer and a Digital Motion Processor in a small 4x4x0.9mm package. The device supports $\mathrm{I} 2 \mathrm{C}$ serial interface and features three 16-bit analog-to-digital converters (ADCs) for digitizing the gyroscope outputs and three 16-bit ADSs for digitizing the accelerometer outputs. An on-chip 1024 Byte FIFO buffer helps lower system power consumption by allowing the system processor to read the sensor data in bursts and then enter a low-power mode as the device collects more data. The device operate from power supply voltage of $2.375 \mathrm{~V}-3.46 \mathrm{~V}$. We used wireless iHealh pulse oximeter to record health rate using dedicated mobile application. The device accurately measure blood oxygen level, pulse rate and perfusion index. It was clinically tested and HIPAA compliant. The device is equipped with wireless Bluetooth 4.0 BLE module, led display system, 3.7V li-ion 390mAh battery and measures pulse rate with $+-2 \mathrm{bpm}$ accuracy or $2 \%$ accuracy. The activity recognition process described in this paper was divided into several steps. In the first step, we implemented in MATLAB algorithms that analyzed the raw data and extracted features. The activity recognition algorithm classified data samples into 2 predefined classes of activities. The first class of activities were chosen to be general: sitting, standing and walking. The second class activity was squatting what is an example of exercising activity. We used supervised classification techniques, therefore the data had to be labeled manually with the appropriate activity during recording and saved in a database. We investigated the following signal features:

(1) Feature extraction in the frequency domain which is the dominant frequency.

(2) Temperature variation.

(3) Heartbeat variation.

All these three features were calculated and collected together in one 3D feature vector. The input signals were filtered using $6^{\text {th }}$ order Butterworth filter. The feature vector was passed through to the classification model, which tried to classify the appropriate class. We recorded 7 volunteers, the model was trained on 5 and tested on the remaining volunteers. We investigated various network topologies. Finally, we applied neural network of the following topology: one neuron has been placed on the output layer, 10 neurons on the hidden layer and 3 neurons in the input layer.

\section{Experimental results and discussion}

We created a dataset that consists of 28 test scenarios. We used the developed Wearable Health Tracking Device (WHTD) and the iHealth wireless pulse oximeter for data acquisition. We analyzed one cohort of healthy volunteers $(\mathrm{n}=7$; mean age $=51 \pm 20.1$ years old $)$. Most of them were women $71.4 \%$ and they were physically active. Volunteers were asked to fasten the WHTD to the lower chest using elastic belt. The temperature sensor was inserted under the arm, while the wireless pulse oximeter was attached to the pointing finger. Volunteers were asked to perform selected daily activities and squatting as an example of rehabilitation exercise according to guided voice commends from computer application. The software recorded the start and the end of each activity from the experiment protocol. The recording was being started when the volunteer was sitting on a chair for 75 seconds, then he stood up and was standing for 75 seconds. Then he was asked to walk. After 60 seconds he stopped and was standing for 15 seconds. After this pause he started exercising for 60 seconds. The volunteer with the WHTD and wireless pulse oximeter was shown in the Fig. 1.

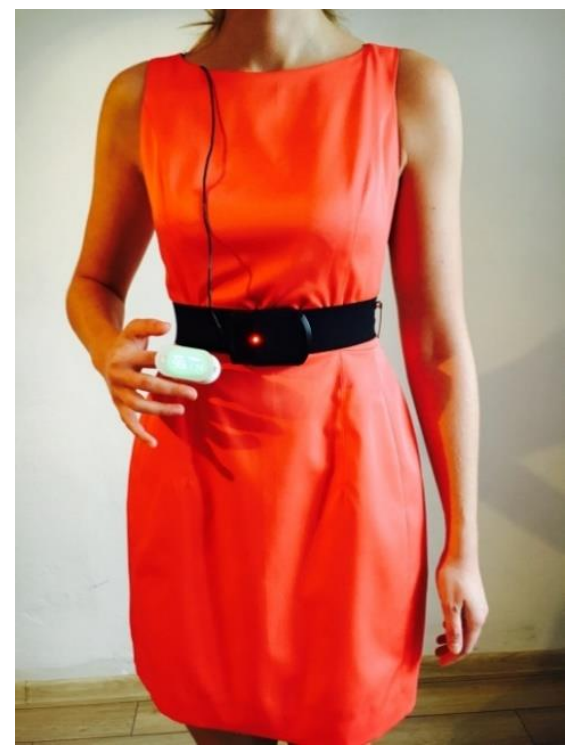

Fig. 1. Volunteer with the WHTD and wireless pulse oximeter.

Data acquired from WHTD for sitting activity performed by 5 volunteers was shown in the Fig. 2 while data acquired for exercising activity completed by 5 volunteers was shown in the Fig. 3. The dataset was analyzed using method described in section 2 . The achieved results were shown in the Table I. 


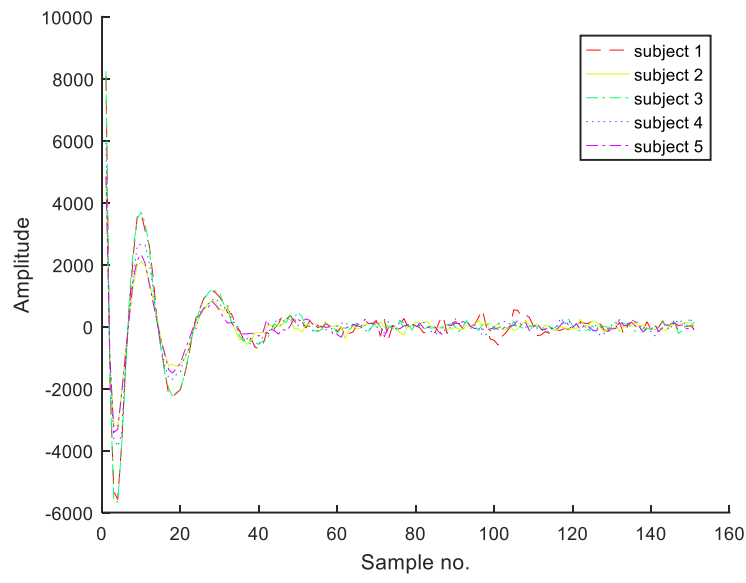

Fig. 2. Sitting.

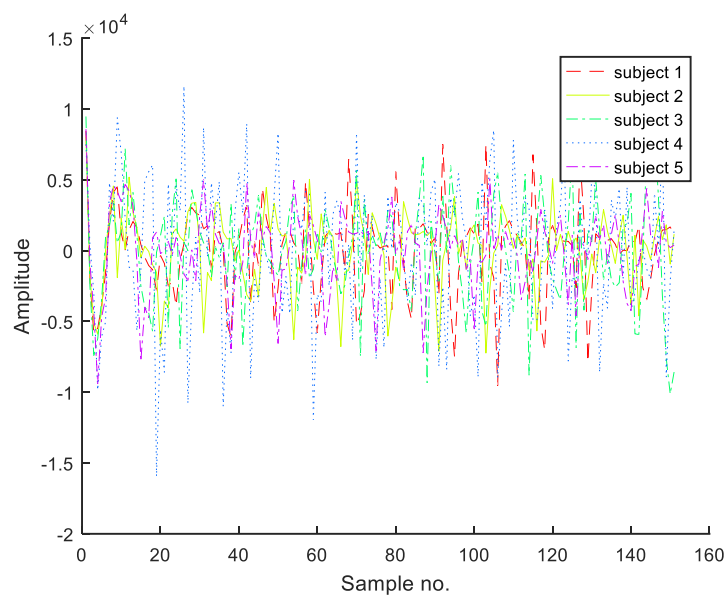

Fig. 3. Exercising.

Table 1. Neural network response results.

\begin{tabular}{cccccccc}
\hline $\begin{array}{c}\text { Activity } \\
\begin{array}{c}\text { Subject } \\
\text { no. }\end{array}\end{array}$ & 1 & 2 & 3 & 4 & 5 & 6 & 7 \\
\hline Sitting & 0,0042 & 0,0045 & 0,0198 & 0,0045 & 0,0065 & 0,0864 & 0,0041 \\
\hline Standing & 0,0940 & 0,0045 & 0,0259 & 0,0042 & 0,0139 & 0,0249 & 0,0682 \\
\hline Walking & 0,0557 & 0,0196 & 0,0006 & 0,0003 & 0,0005 & $\mathbf{0 , 6 2 4 6}$ & $\mathbf{0 , 2 2 7 4}$ \\
\hline $\begin{array}{c}\text { Exercising } \\
\text { Squatting }\end{array}$ & 0,5449 & $\mathbf{0 , 1 8 3 3}$ & 0,9967 & 0,9915 & 0,9649 & 0,9865 & 0,9681 \\
\hline
\end{tabular}

We evaluated the performance of the method using metrics $1-3$.

$$
\begin{gathered}
\text { Accuracy }=\frac{T P+T N}{T P+T N+F P+F N}=82 \% \\
\text { Sensitivity }=\frac{T P}{T P+F N}=85 \%
\end{gathered}
$$

$$
\text { Specificity }=\frac{T N}{T N+F P}=90 \%
$$

According to these statistical characteristics, this method is suited for screening purposes but it is not suitable for the final confirmation of a performed activity. From the results achieved during experiments, we conducted that static activity such as sitting or standing can be easily distinguish from exercising using developed feature vector. Walking was misclassified as exercising in two volunteers. Due to much subject dependant feature variation of walking and squatting using this method we needed to investigate other features that will allow for improved activity differentiation.

\section{Conclusions}

We presented a neural network classification approach for recognition of exercise activity based on the wearable sensor data fusion. We created a dataset of daily activities and exercise activities using developed device. Finally, we implemented and tested recognition system for exercise activity and received $82 \%$ accuracy in free living environment. Developed method can be used in smart home environment especially for people with chronic disease that needs to perform and track specific rehabilitation exercises regularly. Developed method is objective and quantifiable way to track physical rehabilitation. Our ongoing research efforts on recoding more different activates and exercising activities as well as its incorporation into telemedical system.

\section{Acknowledgements}

The scientific work is supported by the AGH University of Science and Technology in year 2017 as a research project No. 11.11.120.612.

\section{References}

[1] Lara OD, Labrador MA. A survey on human activity recognition using wearable sensors. IEEE Communications Surveys and Tutorials. 2013; 15(3), 1192-1209.

[2] Van Kasteren TLM, Englebienne G, Kröse BJ. An activity monitoring system for elderly care using generative and discriminative models. Personal and ubiquitous computing. 2010; 14(6), 489-498.

[3] Del Din S, Godfrey A, Galna B, Lord S, Rochester L. Freeliving gait characteristics in ageing and Parkinson's disease: impact of environment and ambulatory bout length. Journal of NeuroEngineering and Rehabilitation. 2016;13:46. doi:10.1186/s12984-016-0154-5.

[4] Reddy S, Mun M, Burke J, Estrin D, Hansen M, Srivastava M. Using mobile phones to determine transportation modes. ACM Trans. Sensor Networks. 2010; vol. 6, no. 2, 1-27. 
[5] Kańtoch E. Technical verification of applying wearable physiological sensors in ubiquitous health monitoring. Computing in Cardiology 2013; Zaragoza, 269-272.

[6] Kańtoch E. Telemedical human activity monitoring system based on wearable sensors network. In Computing in Cardiology Conference (CinC), IEEE. 2014; 469-472.

[7] Augustyniak P, Kańtoch E. Turning domestic appliances into a sensor network for monitoring of activities of daily living. Journal of Medical Imaging and Health Informatics. 2015; 5(8), 1662-1667.

[8] Kańtoch E. BAN-based health telemonitoring system for inhome care, 2015 Computing in Cardiology Conference (CinC), Nice. 2015; 113-116. doi: 10.1109/CIC.2015.7408599

Address for correspondence.

Eliasz Kańtoch

AGH University of Science and Technology

30 Mickiewicza Av.

30-059 Kraków

Poland.

email:kantoch@agh.edu.pl 